\title{
A test of the diathesis-stress model in the emergency department: Who develops PTSD after an acute coronary syndrome?
}

\author{
Donald Edmondson, PhD, MPH ${ }^{1}$, lan M. Kronish, MD, MPH ${ }^{1}$, Lauren Taggart Wasson, MD, \\ $\mathbf{M P H}^{1}$, James F. Giglio, $\mathbf{M D}^{2}$, Karina W. Davidson, $\mathbf{P h D}^{1}$, and William Whang, $\mathbf{M D}^{1}$ \\ ${ }^{1}$ Center for Behavioral Cardiovascular Health, Department of Medicine, Columbia University \\ Medical Center, New York \\ ${ }^{2}$ Department of Emergency Medicine, Columbia University Medical Center, New York
}

\section{Abstract}

\begin{abstract}
Most acute coronary syndrome (ACS) patients first present to the emergency department (ED). Patients who present to overcrowded EDs develop more posttraumatic stress disorder (PTSD) symptoms due to the ACS than do patients who present to less crowded EDs, but no research has indicated whether some patients may be more vulnerable to the effects of ED crowding than others. In an observational cohort study, we tested whether depressed patients developed more ACS-induced PTSD symptoms under conditions of ED overcrowding than patients who had never been depressed. We conducted psychiatric interviews for current and past depression in 189 ACS patients admitted through the ED within a week of hospitalization, and screened for PTSD symptoms 1 month later using the Impact of Events Scale-Revised. The sum of ED admissions for the 12 hours prior to and 12 hours after each participant's admission was categorized into tertiles for analysis. In a 3 (ED crowding tertile) by 3 (never, past, current depression) analysis of covariance adjusted for demographic and clinical factors, we found significant effects for ED crowding, depression status, and their interaction (all p's <.05). Mean PTSD scores were significantly higher $(\mathrm{p}=.005)$ for participants who were currently depressed and were treated during times of high ED crowding [25.38, 95\% CI= 16.18-34.58], or had a history of depression [10.74, 95\% CI= 6.86-14.62], relative to all other participants, who scored 5.6 or less. These results suggest that depressed ACS patients may be most vulnerable to the stress-inducing effects of ED crowding.
\end{abstract}

An acute coronary syndrome (ACS; myocardial infarction or unstable angina) is a highly stressful experience that leads as many as 1 in 8 ACS patients to subsequently develop

\footnotetext{
(c) 2014 Elsevier Ltd. All rights reserved.

Correspondence: Donald Edmondson, Ph.D., M.P.H., Center for Behavioral Cardiovascular Health, Columbia University Medical Center, 622 West 168 Street, PH9-317, New York, NY 10032; dee2109@ columbia.edu; phone: 212-342-3674; fax: 212-305-3172. Conflict of interest: The authors have no conflicts of interest to report.

Publisher's Disclaimer: This is a PDF file of an unedited manuscript that has been accepted for publication. As a service to our customers we are providing this early version of the manuscript. The manuscript will undergo copyediting, typesetting, and review of the resulting proof before it is published in its final citable form. Please note that during the production process errors may be discovered which could affect the content, and all legal disclaimers that apply to the journal pertain.
} 
posttraumatic stress disorder (PTSD) (Edmondson et al., 2012b; Gander \& von Kanel, 2006). Not only does PTSD significantly impact quality of life, but it is also associated with a doubling of risk for recurrent ACS and mortality in the first 3 years after the index ACS event (Edmondson \& Cohen, 2013a; Edmondson et al., 2012b; Edmondson et al., 2011; von Känel et al., 2011).

Most ACS patients present first to the emergency department (ED) (Kantor, Glassman, Bigger, Perel, \& Giardina, 1978), and the ED to which they present is often overcrowded and chaotic (Hoot \& Aronsky, 2008). Indeed, ED overcrowding is a problem, at least intermittently, in most hospitals throughout the US (Akhtar, Husk, Waxman, \& Sonara, 2004; Handel et al., 2011). Recently, we showed that overcrowding in the ED during ACS treatment is independently associated with greater PTSD symptoms due to the ACS event at one month after hospital discharge (Edmondson, Shimbo, Ye, Wyer, \& Davidson, 2013b). Given the adverse impact of PTSD on medical and psychiatric outcomes, it is essential to understand which ACS patients are most vulnerable to the stress-inducing effects of ED overcrowding.

Depression appears to increase PTSD risk across traumatic event types (Blanchard, Hickling, Taylor, Loos, \& Gerardi, 1994; Resnick, Kilpatrick, Best, \& Kramer, 1992), but retrospective reporting on pre-trauma depression and the high comorbidity of depression and PTSD after traumatic events has posed difficulties for determining causality. Further, it is unclear how depression influences participants' self-report of the stressfulness of a given potentially traumatic event. Previous research is mixed on whether depressed patients are more psychologically reactive than non-depressed patients to objectively identical stressors (Burke, Davis, Otte, \& Mohr, 2005). However, a systematic review of more than 20 studies showed that in comparison with non-depressed individuals, depressed individuals have exaggerated heart rate and blood pressure responses to laboratory stressors (Jørgensen, Nakayama, Reith, Raaschou, \& Olsen, 1997).

The diathesis-stress model of PTSD states that preexisting vulnerabilities interact with life stress to produce psychological disorder (McKeever \& Huff, 2003). That is, individuals who have some diathesis for the development of PTSD, due to some genetic, biological, or psychosocial liability, are at high risk for developing that disorder when the degree of external stress to which they are exposed crosses the threshold required to activate the diathesis. To test the diathesis-stress model of PTSD in ACS patients, we estimated the potential association of pre-trauma depression, a particularly stressful aspect of the ACS experience, and subsequent PTSD. If pre-trauma depression is a vulnerability factor for subsequent PTSD, and the diathesis-stress model is correct, then depression we expect to see a moderator effect such that depression is strongly associated with subsequent PTSD symptoms when ACS patients are treated under conditions of high stress (ie, high ED crowding). If depression is a vulnerability for subsequent PTSD, but the diathesis-stress model is incorrect, we would expect significant main effects for pre-event depression and high ED crowding, as independent causes of subsequent PTSD symptoms, but no moderator effect. A seminal study in this area (Resnick et al., 1992) found support for the diathesisstress model in crime victims, wherein those who retrospectively reported pre-trauma depression had significantly greater current PTSD symptoms-but only for those who self- 
reported high crime stress (ie, injury during the crime, and high perceived life threat). In the present study, we tested the diathesis-stress model of PTSD due to ACS, by estimating the associations among pre- and peri-event depression measured at the time of the ACS event and exposure to ED crowding (an objective real-world assessment of the stressfulness of a potentially traumatic event) with subsequent PTSD symptoms in ACS patients.

\section{Methods}

\section{Ethics statement}

The Institutional Review Board of Columbia University approved this study, and all participants provided informed consent.

\section{Study design and participants}

Participants were hospitalized ACS patients with enrolled in the Prescription Use, Lifestyle, and Stress Evaluation (PULSE) study, an ongoing, single site, prospective, observational cohort study of the prognostic risk conferred by psychosocial factors at the time of an ACS. Patients with acute ST segment elevation MI (STEMI), non-ST segment elevation myocardial infarction (NSTEMI), or unstable angina pectoris (UA) were recruited from Columbia University Medical Center within one week of hospitalization for their ACS and completed a self-report depression screen at enrollment. At 3-7 days post-discharge, patients completed a structured psychiatric interview by telephone for depression diagnosis. At 1 month post-discharge, participants were interviewed by telephone to screen for ACSinduced PTSD and depression symptoms. All study personnel were blind to ED crowding status. The current analyses include only participants who presented to Columbia University Medical Center's ED and completed the ACS-induced PTSD interview. Excluded from analyses were participants who were enrolled prior to initiation of PTSD data collection $(n=$ 212). Compared to the participants in this study, the excluded participants did not differ on baseline depression or any demographic or clinical covariates (all p's $>.10$ ). Data collection occurred between 2009 and 2011.

Measures

ACS-induced PTSD—We assessed ACS-induced PTSD symptoms by telephone interview 1 month after discharge using the Impact of Events Scale- Revised (IES-R)(Weiss, 1997) -specific for ACS. Participants used a 5-point scale ranging from 0 ("not at all") to 4 ("extremely") to report the extent to which they had been bothered by each of 22 PTSD symptoms in the past week with reference to their hospitalization for ACS. Specifically, they were told, "Think about when you were hospitalized on [DATE], and we enrolled you in this study. In the past 7 days have you been bothered by any of the following in reference to that hospitalization?" The IES-R yields a total score, and the internal validity of this score in the current study was acceptable (Cronbach's $a=0.94)$.

ED crowding-Based on our previous research, and our observation that the mean length of stay (LOS) in the ED in this sample was $>11$ hours, we calculated the degree of ED crowding exposure for each participant by summing the hourly ED admissions for the 12 hours prior to and the 12 hours subsequent to each participant's ED presentation time, and 
we divided the score into tertiles for analysis.(Edmondson et al., 2013b), While no gold standard measure for ED crowding measurement currently exists, similar approaches to measuring ED crowding have been used in prior studies (Higginson, 2012).

In-hospital depression screening measure-We screened for in-hospital depressive symptoms using the Patient Health Questionnaire-9 (PHQ-9) (Kroenke, Spitzer, \& Williams, 2001), which measures the frequency of depressive symptoms according to the criteria for depression according to the Diagnostic and Statistical Manual of Mental Disorders Fourth Edition (American Psychiatric Association, 1994). Using a cutpoint of 10, the instrument has a sensitivity of $91 \%$ and specificity of $89 \%$ for diagnosing major depression when compared with a structured interview performed by a mental health professional (Williams et al., 2005).

Depression diagnosis-Within 7 days of hospital admission, participants underwent a psychiatric interview by telephone to assess major depressive disorder (MDD). We used the Diagnostic Interview Schedule with Hamilton Ratings (DISH), a semistructured diagnostic interview, to diagnose MDD (Freedland et al., 2002). The DISH is based on a modified version of the National Institute of Mental Health Diagnostic Interview Schedule. It was developed to assess comorbid MDD in medically ill patients, and has been used in some of the most important studies of depression in post-MI and other cardiac populations, including the Enhancing Recovery in Coronary Heart Disease study (Berkman et al., 2003). When trained interviewers use this interview, and quality assurance is conducted, the concordance with other structured psychiatric interviews is excellent (Freedland et al., 2002). After the initial psychiatric interview, participants were then asked: "Have you ever been depressed before?" If participants said yes or were uncertain, they were then probed further to estimate whether they had fulfilled Diagnostic and Statistical Manual of Mental Disorders, $4^{\text {th }}$ Edition (DSM-IV) criteria for a positive history of MDD (American Psychiatric Association, 1994). This was evaluated by assessing whether a depressive episode meeting DSM criteria lasted at least 2 weeks, and was sufficiently severe to affect functioning. Patients with a diagnosis of MDD at time of the interview were classified as "current depression," regardless of depression history. Patients with a positive history of MDD but who were not currently depressed were classified as "past depression." Participants with neither current nor past depression were classified as "never depressed."

Depression symptoms at 1 month post-ACS-We assessed depression symptoms concurrently with PTSD symptoms at 1 month post-ACS using the Beck Depression Inventory (BDI)(Beck, Ward, Mendelson, Mock, \& Erbaugh, 1961). The BDI is a 21-item self-report instrument with questions that address the frequency/severity of depressive symptoms during the previous week. Patients rate the extent to which each symptom is present or absent using a 4-point scale ranging from 0 to 3 for a total score of 0 to 63 .

Demographic covariates-We assessed participants' age, sex, race (Black/African American=1, White or other $=0$ ), and ethnicity (Hispanic=1, non-Hispanic=0) by self-report.

ACS type-A study cardiologist used standard criteria (Cannon et al., 2001) to determine ACS diagnosis type from the medical record (Unstable Angina=0, NSTEMI=1, STEMI=2). 
Global Registry of Acute Coronary Events (GRACE) risk score (Eagle et al., 2004)-The GRACE index is a postdischarge prediction model for 6-month mortality in patients with cardiac disease derived from a multinational registry (Eagle et al., 2004). The variables collected from the medical record in the GRACE index are age, history of MI, history of heart failure, presenting pulse rate, systolic blood pressure at presentation, initial serum creatinine level, initial cardiac enzyme levels, ST-segment depression on presenting electrocardiogram, and in-hospital percutaneous coronary intervention. The GRACE index has a range from 1 to 263 points with higher scores indicating greater mortality risk.

\section{Charlson Comorbidity Index (Charlson, Szatrowski, Peterson, \& Gold, 1994)-}

We abstracted the 19 conditions that are included in the Charlson comorbidity index (eg, congestive heart failure, diabetes mellitus) from the medical record. To calculate the Charlson index, conditions are weighted from 0 to 6 , and points are then summed to generate a total score that can range from 0 to 37 . This overall score reflects cumulative increased likelihood of 1-year mortality; the higher the score, the more severe the comorbidity.

Statistical analysis-We tested for differences on demographic, clinical, and in-hospital depression variables across ED crowding tertiles and depression status using one-way analysis of variance (ANOVA) for continuous variables and chi-square for categorical variables. To test whether patient depression is a vulnerability for the development of PTSD symptoms under conditions of greater ED crowding, we conducted a 3 (ED crowding status: low vs. medium vs. high) X 3 (depression status by diagnosis: never depressed vs. past depression only vs. current depression) analysis of covariance (ANCOVA) with IES-R score at 1 month post-discharge as the dependent variable. Covariates included age, sex, Black race, Hispanic ethnicity, ACS type, GRACE cardiac risk score (an index of severity), and Charlson comorbidity index, all of which have been implicated in the development of psychiatric disorder after acute cardiovascular events (Edmondson et al., 2013a). In a post hoc analysis, we conducted the same analysis except that we replaced the depression diagnosis factor with a 2-level depression screen factor (positive vs. negative screen).

\section{Results}

The 189 ACS patients who participated in the study are described in Table 1. Fourteen participants (7.4\%) were diagnosed with current depression, 59 (31.2\%) with past depression, and 116 (61.4\%) were never depressed. Women were more likely to be depressed or have a past history of depression ( $\mathrm{p}<.05$ ), but depression groups did not differ on any other demographic or clinical characteristics. ED census, and tertile of ED crowding, was distributed equally across depression groups. Currently depressed participants had higher PHQ ( $<<.001)$ and IES-R ( $<<.05)$ scores than past or never depressed participants, but notably only $37 \%$ of participants who were diagnosed with depression screened positive for depression on the PHQ.

Results of the primary analysis showed that the effects of ED crowding, depression status, and their interaction on subsequent PTSD symptoms were significant (Figure 1). There was a main effect of ED crowding, $F(2,173)=11.34, p<.001$, partial $\eta^{2}=.12$, such that 
participants treated during times when the ED was most crowded scored significantly higher $(\mathrm{p}<.001)$ on the PTSD scale at 1 month [(IES-R mean score of $13.68(95 \% \mathrm{CI}=$ 10.22-17.14)], than the those treated during the lowest or medium tertiles of ED crowding [IES-R scores $3.44(95 \% \mathrm{CI}=0.15-6.73)$ and 3.60 (95\% CI= 0.40-6.80), respectively].

There was also a main effect of depression, $F(2,173)=4.45, p=.01$, partial $\eta^{2}=.05$, such that participants who were currently depressed during hospitalization reported significantly greater $(\mathrm{p}=.02)$ PTSD symptoms at 1 month [IES-R mean score of $12.05(95 \% \mathrm{CI}=$ 7.14-16.96)] relative to participants who had never been depressed or who had a positive history of depression [IES-R mean score $4.33(95 \% \mathrm{CI}=2.63-6.03)$ and 4.34 (95\% CI= 1.93-6.76), respectively].

Finally, there was a significant 2-way ED crowding $\mathrm{X}$ depression interaction, $F(4,173)=$ $3.85, p=.005$, partial $\eta^{2}=.08$. The interaction was such that participants who were currently depressed during hospitalization and were treated during times when the ED was most crowded reported the highest 1-month PTSD score (25.38, 95\% CI=16.18-34.58), and those with a history of depression treated during times when the ED was most crowded reported the second highest 1-month PTSD score (10.74, 95\% CI=6.86-14.62). The other ED crowding/depression groups reported 1-month PTSD scores less than 5.6, and they did not differ significantly from each other.

\section{Post hoc analyses}

Potential effect of ED crowding on depression diagnosis-Since depression diagnoses were made after participants had been exposed to ED treatment, we tested whether the degree of ED crowding they were exposed to influenced their likelihood of being diagnosed with current depression or past depression. ED crowding tertiles were not associated with depression diagnosis, $\chi^{2}(4)=2.77, \mathrm{p}=.60$, or with PHQ scores, $r=-.05, \mathrm{p}=$. 31.

The influence of ED length of stay (LOS)—Our previous research suggested that ED LOS is not associated with subsequent ACS-induced PTSD symptoms (Edmondson et al., 2013b), but may be associated with depression (Edmondson, Newman, Chang, Wyer, \& Davidson, 2012a). To determine whether ED LOS was associated with PTSD symptoms or the interaction of depression and ED crowding, we tested whether ED LOS was associated with ED crowding, depression, or PTSD symptoms. To calculate ED LOS, we took the difference between time of ED triage recorded in the medical record and the time of transfer to an inpatient bed. ED LOS was not associated with ED crowding, depression (diagnosis or PHQ score), or PTSD symptoms (all p's >.34).

The utility of in-hospital depression screening for determining PTSD risk-The results of the primary analysis suggest that a clinical diagnosis of depression or a history of depression as determined by a clinician are useful for determining risk for PTSD symptoms after an ACS. However, as those methods for determining patient depression status are not useful in most clinical settings, we tested whether a brief in-hospital self-report screen for depression functions similarly. We conducted a 3 (ED crowding status: high vs. medium vs. 
low) X 2 (PHQ score $\geq 10$ vs. PHQ score < 10) analysis of covariance (ANCOVA), with the same covariates as the primary analysis.

Similar to the primary analysis, results suggested a main effect of ED crowding, $F(2,175)=$ $18.57, p<.001$, partial $\eta^{2}=.18$, and a main effect of PHQ score, $F(1,175)=11.63, p<.001$, partial $\eta^{2}=.06$. Most importantly, there was a significant 2-way ED crowding X BDI score interaction effect, $F(2,175)=12.10, p<.001$, partial $\eta^{2}=.12$, such that participants who scored $\geq 10$ on the PHQ during hospitalization and were treated during times when the ED was most crowded scored 4 times greater on the PTSD scale at 1 month relative to all other participants. The mean 1-month PTSD score for participants who screened positive for depression and were treated when the ED was most crowded was $26.97(95 \% \mathrm{CI}=$ 20.30-33.64), while the mean score in all other cells (which did not differ from one another) was 5.89 or less.

Do depressed patients exposed to ED crowding experience more depression symptoms at 1 month?-Elevated depression symptoms are common after highly stressful or traumatic events (Galea et al., 2002), and genetic factors common to both depression and PTSD have been reported (Koenen et al., 2008). As such, we tested whether depression may be a vulnerability not only for the development of PTSD symptoms, but also for increased depression symptoms, under conditions of high ED crowding during ACS treatment. We tested the same model as the primary analysis, except that we replaced the 1month IES score with the 1-month BDI score as the dependent variable. As expected, there was a main effect of depression status on 1-month BDI score ( $p<.001)$, but there was no effect of ED crowding ( $\mathrm{p}=.09)$ and no significant interaction of depression status and ED crowding $(\mathrm{p}=.17)$.

\section{Discussion}

Our previous research showed that ED crowding during treatment for ACS was associated with greater subsequent PTSD symptoms, but did not give clinicians any indication as to which patients are most vulnerable to the psychological effects of ED crowding. This study's results suggest that the effect of ED crowding on subsequent PTSD symptoms is greatly magnified for patients who are depressed when the ACS event occurs. Indeed, the mean PTSD score in the currently depressed patients treated during times of high ED crowding was greater than the suggested cutoff score for clinically significant PTSD symptoms after ACS, (Rocha et al., 2008), whereas those who were never depressed developed few PTSD symptoms regardless of how crowded the ED was during their treatment.

These results offer support for the diathesis-stress model of PTSD in ACS patients. Whereas depressed patients treated during times of low ED census were not significantly different from patients who were not depressed, it appears that the added stress of ED overcrowding seems to have markedly impacted depressed patients' risk for development of PTSD symptoms. This may be because overcrowding in the ED differentially impacts depressed patients' perception of the ACS event, making it potentially more traumatic and leading to greater subsequent PTSD symptoms. As such, depression appears to be a vulnerability factor for subsequent PTSD, but only under circumstances of high objective stress. Future research 
should test this hypothesis by assessing whether increased perceptions of stress during a stay in overcrowded EDs occur specifically in depressed patients.

Even in the absence of current depression diagnosis, patients who have been depressed in the past appear more vulnerable to the lasting psychological effects of ED crowding than patients who have never been depressed. These findings suggest that the increased psychological and physiological reactivity to stressors that has been shown in otherwise healthy depressed patients may persist once the major depressive episode has remitted, and may be responsible for the interactive effect of ED crowding and past depression on ACSinduced PTSD symptoms.

While clinical diagnosis is the gold standard for identifying MDD, in this study, a self-report depression screening instrument that is already widely used in cardiac populations was able to identify patients who were psychologically vulnerable to ED crowding. This suggests that screening for depression in ACS patients in the ED could help to direct stress-reduction efforts during times of high ED crowding. For example, a recent study found that patientdirected music intervention delivered with noise-cancelling headphones significantly reduced anxiety and both sedation frequency and intensity in patients receiving mechanical ventilator support (Chlan et al., 2013). Similar stress-reduction techniques should be tested in vulnerable ACS patients who often wait for multiple hours in overcrowded EDs. Further, there is little reason to believe that the interactive effect of depression and ED crowding on PTSD found in this study is a phenomenon specific to ACS patients. ACS is only one of many potentially traumatic experiences faced by patients in the ED, and as many as $20 \%$ of all ED patients screen positive for depression (Hoyer \& David, 2008). Future research should determine whether all depressed patients facing life-threatening circumstances in overcrowded EDs are at increased risk for PTSD symptoms.

\section{Limitations}

The first limitation of this study is the use of a self-report screening instrument for ACSinduced PTSD symptoms, though scores on the instrument we used—and others like itidentify ACS recurrence and mortality risk in ACS patients (Edmondson et al., 2013a; Edmondson et al., 2012b; Edmondson et al., 2011). A related issue is that we assessed PTSD symptoms with reference to participants' ACS "hospitalization," and many aspects of an ACS event that occur prior to hospitalization may be traumatic. We chose this language because we wanted to insure that participants were answering with reference to the specific ACS event that prompted their enrollment in the study, as opposed to their heart problems in general, any ACS event they may have experienced in the past, or symptoms they experienced since their hospitalization. Based on our previous research using similar wording, we believe that this strategy is valid for assessing PTSD due to an ACS event.

The study's second limitation is the lack of assessment of participants' reactivity to stress in the ED. We believe that our findings suggest that depressed patients are more reactive to the stress of ED overcrowding, which causes the event to be perceived as more traumatic, and leads to greater PTSD symptoms in the future, but we were not able to test this hypothesis. The third limitation is that these analyses were conducted on data from a small, albeit racially and ethnically diverse, sample of patients treated in an urban teaching hospital with 
a very busy ED. As such, these results may not generalize to patients or hospitals with different characteristics.

\section{Theoretical considerations}

While the primary focus of this paper is an applied one--the identification of ACS patients at risk for future PTSD—we believe that our findings are of theoretical importance for researchers and clinicians interested in the role of depression in psychological responses to traumatic events more broadly. A great deal of research has been devoted to the comorbidity of depression and PTSD, but the unpredictability of traumatic events has made prospective studies of their temporal association less common. Even so, our results support a diathesisstress model of PTSD, and closely mirror those of early research on depression as a vulnerability factor for PTSD (Resnick et al., 1992). Further, we believe these results point to increased cognitive stress reactivity in depression as a potential target for PTSD prevention. Finally, it is important to note that most ACS patients (including most depressed individuals) are resilient (Bonanno, 2004), but at high levels of stress, the vulnerability associated with pre-existing depression exerts its influence.

\section{Conclusions}

As many as $20 \%$ of ACS patients meet criteria for MDD during hospitalization, and up to 47\% have significant patient-reported depressive symptoms (Bush et al., 2005; Carney \& Freedland, 2008). This study suggests that if those patients are treated in overcrowded EDs, they are at substantially increased risk for subsequently developing PTSD due to the ACS event. Future research should focus on interventions to reduce ED crowding and patient stress in the ED, and those interventions should be targeted first at patients who are, or have been, depressed.

\section{Acknowledgments}

This work was supported by grants HL-088117 and HL-117832 from the National Heart, Lung, and Blood Institute (NHLBI) and in part by Columbia University's CTSA grant No.UL1RR024156 from NCATS-NCRR/National Institutes of Health, Bethesda, Maryland, United States. Dr. Kronish was supported by grant K23-HL098359 from NHLBI. . The contents of this manuscript are solely the responsibility of the authors and do not necessarily represent the official view of the National Center for Research Resources or the National Institutes of Health.

\section{References}

Akhtar S, Husk G, Waxman D, Sonara P. Emergency department crowding: A national perspective. Annals of Emergency Medicine. 2004; 44:S28.

American Psychiatric Association. Diagnostic and statistical manual of mental disorders. 4th. Washington, D.C.: American Psychiatric Association; 1994.

Beck AT, Ward CH, Mendelson M, Mock J, Erbaugh J. An inventory for measuring depression. Arch Gen Psychiatry. 1961; 4:561-571. [PubMed: 13688369]

Berkman LF, Blumenthal J, Burg M, Carney RM, Catellier D, Cowan MJ, Czajkowski SM, DeBusk R, Hosking J, Jaffee A. Effects of treating depression and low perceived social support on clinical events after myocardial infarction: the Enhancing Recovery in Coronary Heart Disease Patients (ENRICHD) Randomized Trial. JAMA: Journal of the American Medical Association. 2003

Blanchard EB, Hickling EJ, Taylor AE, Loos WR, Gerardi RJ. Psychological morbidity associated with motor vehicle accidents. Behaviour Research and Therapy. 1994; 32:283-290. [PubMed: 8192626] 
Bonanno GA. Loss, trauma, and human resilience: Have we underestimated the human capacity to thrive after extremely aversive events? American Psychologist. 2004; 59:20. [PubMed: 14736317]

Burke HM, Davis MC, Otte C, Mohr DC. Depression and cortisol responses to psychological stress: A meta-analysis. Psychoneuroendocrinology. 2005; 30:846-856. [PubMed: 15961250]

Bush, DE.; Ziegelstein, RC.; Patel, UV.; Thombs, BD.; Ford, DE.; Fauerbach, JA.; McCann, UD.; Stewart, KJ.; Tsilidis, KK.; Patel, AL.; Feuerstein, CJ.; Bass, EB. Post-myocardial Infarction Depression Summary, Evidence Report/Technology Assessment. Agency for Healthcare Research and Quality; 2005. p. 1-8.

Cannon CP, Battler A, Brindis RG, Cox JL, Ellis SG, Every NR, Flaherty JT, Harrington RA, Krumholz HM, Simoons ML, Van De Werf FJ, Weintraub WS, Mitchell KR, Morrisson SL, Anderson HV, Cannom DS, Chitwood WR, Cigarroa JE, Collins-Nakai RL, Gibbons RJ, Grover FL, Heidenreich PA, Khandheria BK, Knoebel SB, Krumholz HL, Malenka DJ, Mark DB, McKay CR, Passamani ER, Radford MJ, Riner RN, Schwartz JB, Shaw RE, Shemin RJ, Van Fossen DB, Verrier ED, Watkins MW, Phoubandith DR, Furnelli T. American College of Cardiology key data elements and definitions for measuring the clinical management and outcomes of patients with acute coronary syndromes. A report of the American College of Cardiology Task Force on Clinical Data Standards (Acute Coronary Syndromes Writing Committee). Journal of the American College of Cardiology. 2001; 38:2114-2130. [PubMed: 11738323]

Carney RM, Freedland KE. Depression in patients with coronary heart disease. American Journal of Medicine. 2008; 121:S20-27. [PubMed: 18954589]

Charlson M, Szatrowski T, Peterson J, Gold J. Validation of a combined comorbidity index. Journal of Clinical Epidemiology. 1994; 47:1245-1251. [PubMed: 7722560]

Chlan LL, Weinert CR, Heiderscheit A, Tracy MF, Skaar DJ, Guttormson JL, Savik K. Effects of Patient-Directed Music Intervention on Anxiety and Sedative Exposure in Critically Ill Patients Receiving Mechanical Ventilatory SupportA Randomized Clinical TrialPatient-Directed Music Intervention. JAMA. 2013; 309:2335-2344. [PubMed: 23689789]

Eagle KA, Lim MJ, Dabbous OH, Pieper KS, Goldberg RJ, Van de Werf F, Goodman SG, Granger CB, Steg PG, Gore JM, Budaj A, Avezum A, Flather MD, Fox KA. A validated prediction model for all forms of acute coronary syndrome: estimating the risk of 6-month postdischarge death in an international registry. JAMA. 2004; 291:2727-2733. [PubMed: 15187054]

Edmondson D, Cohen BE. Posttraumatic stress disorder and cardiovascular disease. Prog Cardiovasc Dis. 2013a; 55:548-556. [PubMed: 23621964]

Edmondson D, Newman JD, Chang MJ, Wyer P, Davidson KW. Depression is associated with longer emergency department length of stay in acute coronary syndrome patients. BMC Emerg Med. 2012a; 12:14. [PubMed: 23126447]

Edmondson D, Richardson S, Falzon L, Davidson KW, Mills MA, Neria Y. Posttraumatic stress disorder prevalence and risk of recurrence in acute coronary syndrome patients: A meta-analytic review. PLoS One. 2012b; 7:e38915. [PubMed: 22745687]

Edmondson D, Rieckmann N, Shaffer JA, Schwartz JE, Burg MM, Davidson KW, Clemow L, Shimbo D, Kronish IM. Posttraumatic stress due to an acute coronary syndrome increases risk of 42-month major adverse cardiac events and all-cause mortality. J Psychiatr Res. 2011; 45:1621-1626. [PubMed: 21807378]

Edmondson D, Shimbo D, Ye S, Wyer P, Davidson KW. The association of emergency department crowding during treatment for acute coronary syndrome with subsequent posttraumatic stress disorder symptoms. JAMA Internal Medicine. 2013b; 173:472-474. [PubMed: 23400256]

Freedland KE, Skala JA, Carney RM, Raczynski JM, Taylor CB, de Leon CFM, Ironson G, Youngblood ME, Krishnan KRR, Veith RC. The Depression Interview and Structured Hamilton (DISH): rationale, development, characteristics, and clinical validity. Psychosomatic Medicine. 2002; 64:897-905. [PubMed: 12461195]

Galea S, Ahern J, Resnick H, Kilpatrick D, Bucuvalas M, Gold J, Vlahov D. Psychological sequelae of the September 11 terrorist attacks in New York City. New England Journal of Medicine. 2002; 346:982-987. [PubMed: 11919308]

Gander ML, von Kanel R. Myocardial infarction and post-traumatic stress disorder: frequency, outcome, and atherosclerotic mechanisms. European Journal of Cardiovascular Prevention \& Rehabilitation. 2006; 13:165-172. [PubMed: 16575268] 
Handel DA, Pines J, Aronsky D, Genes N, Ginde AA, Hackman J, Hilton JA, Hwang U, Kamali M, Powell E, Sattarian M, Fu R. Variations in Crowding and Ambulance Diversion in Nine Emergency Departments. Academic Emergency Medicine. 2011; 18:941-946. [PubMed: 21906203]

Higginson I. Emergency department crowding. Emergency medicine journal. 2012

Hoot N, Aronsky D. Systematic review of emergency department crowding: causes, effects, and solutions. Annals of Emergency Medicine. 2008; 52:126-136. [PubMed: 18433933]

Hoyer D, David E. Screening for depression in emergency department patients. The Journal of emergency medicine. 2008

Jørgensen H, Nakayama H, Reith J, Raaschou H, Olsen T. Stroke recurrence: predictors, severity, and prognosis. The Copenhagen Stroke Study Neurology. 1997; 48:891.

Kantor SJ, Glassman AH, Bigger JT Jr, Perel JM, Giardina EV. The cardiac effects of therapeutic plasma concentrations of imipramine. American Journal of Psychiatry. 1978; 135:534-538. [PubMed: 645944]

Koenen KC, Fu QJ, Ertel K, Lyons MJ, Eisen SA, True WR, Goldberg J, Tsuang MT. Common genetic liability to major depression and posttraumatic stress disorder in men. Journal of Affective Disorders. 2008; 105:109-115. [PubMed: 17540456]

Kroenke K, Spitzer RL, Williams JB. The PHQ-9: validity of a brief depression severity measure. J Gen Intern Med. 2001; 16:606-613. [PubMed: 11556941]

McKeever, VM.; Huff, ME. A diathesis-stress model of posttraumatic stress disorder: Ecological, biological, and residual stress pathways. US: Educational Publishing Foundation; 2003. p. 237-250.

Resnick HS, Kilpatrick DG, Best CL, Kramer TL. Vulnerability-stress factors in development of posttraumatic stress disorder. The Journal of Nervous and Mental Disease. 1992; 180:424-430. [PubMed: 1624923]

Rocha LP, Peterson JC, Meyers B, Boutin-Foster C, Charlson ME, Jayasinghe N, Bruce ML. Incidence of posttraumatic stress disorder (PTSD) after myocardial infarction (MI) and predictors of ptsd symptoms post-MI--a brief report. International Journal of Psychiatry in Medicine. 2008; 38:297-306. [PubMed: 19069574]

von Känel R, Hari R, Schmid JP, Wiedemar L, Guler E, Barth J, Saner H, Schnyder U, Begré S. Nonfatal cardiovascular outcome in patients with posttraumatic stress symptoms caused by myocardial infarction. Journal of Cardiology. 2011; 58:61-68. [PubMed: 21493042]

Weiss D. The impact of event scale: Revised. Cross-Cultural Assessment of Psychological Trauma and PTSD. 1997:219-238.

Williams LS, Brizendine EJ, Plue L, Bakas T, Tu W, Hendrie H, Kroenke K. Performance of the PHQ-9 as a screening tool for depression after stroke. Stroke. 2005; 36:635-638. [PubMed: 15677576] 


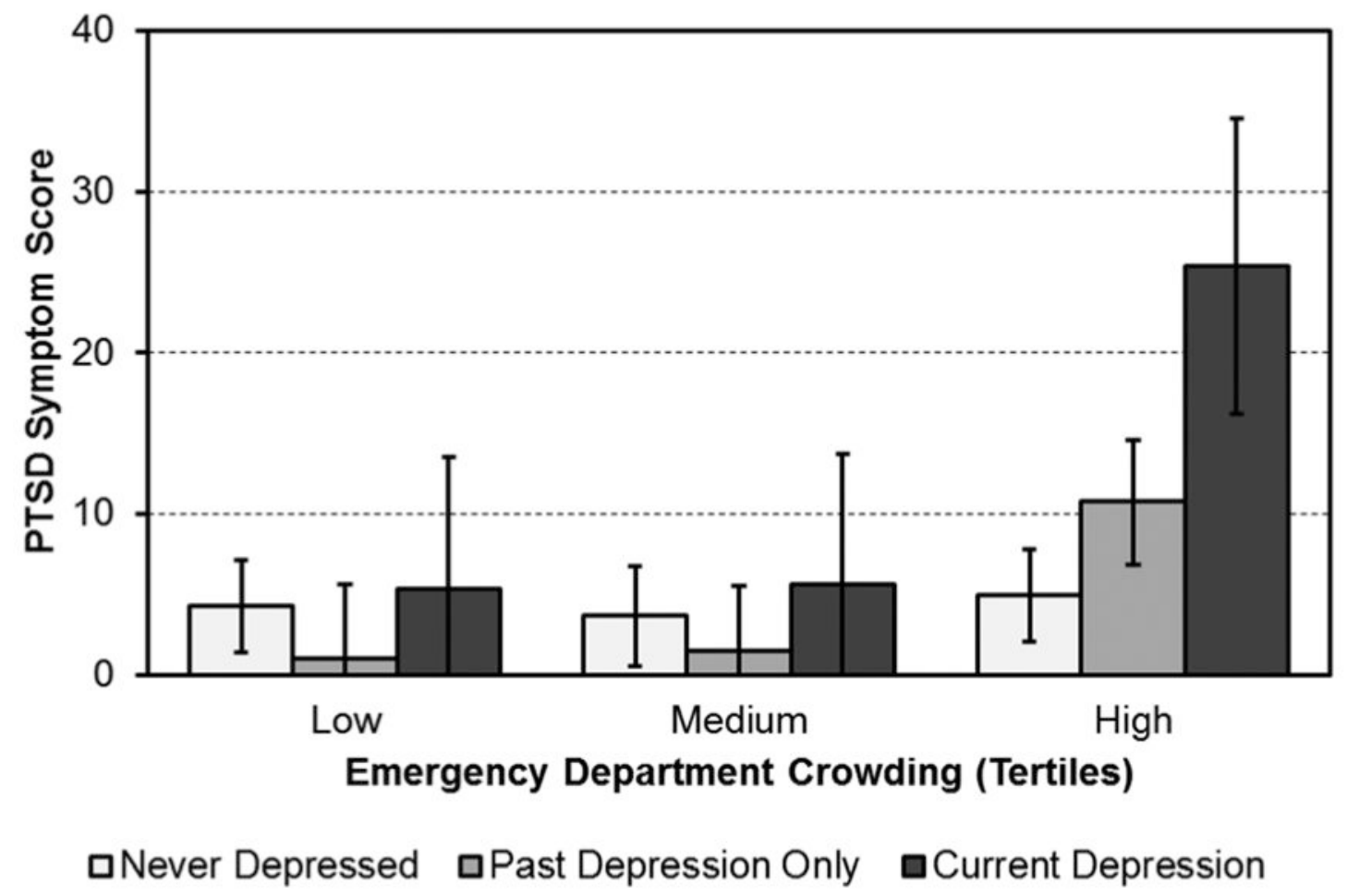

Figure 1.

ACS-induced PTSD symptoms at 1 month after discharge by ED crowding tertile and depression diagnosis status. 


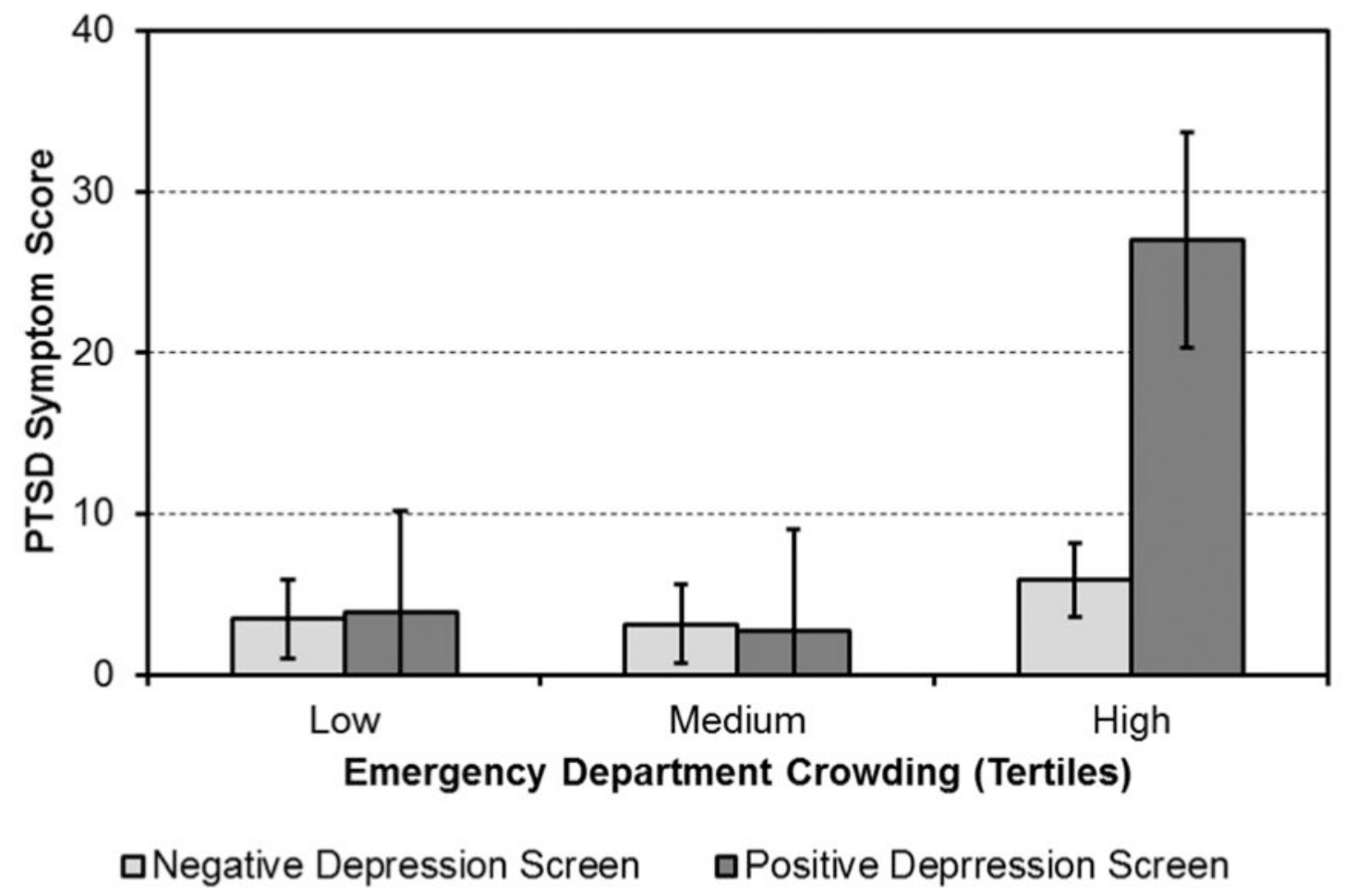

Figure 2.

ACS-induced PTSD symptoms at 1 month after discharge by ED crowding tertile and depression screen. 
Table

Participant Characteristics by Depression Status

\begin{tabular}{|c|c|c|c|}
\hline Characteristic & $\underset{116)}{\text { Never Depressed }(\mathrm{N}=}$ & $\begin{array}{l}\text { Past Depression }(\mathrm{N}= \\
\text { 59) }\end{array}$ & $\begin{array}{l}\text { Current Depression } \\
(\mathrm{N}=\mathbf{1 4})\end{array}$ \\
\hline \multicolumn{4}{|l|}{ Demographic } \\
\hline Age, y & $62.7 \pm 11.1$ & $63.1 \pm 11.1$ & $62.5 \pm 10.9$ \\
\hline Male, $n(\%)^{*}$ & $89(76)$ & $38(64)$ & $7(50)$ \\
\hline Black/African American, $n(\%)$ & $29(25)$ & $12(20)$ & $5(36)$ \\
\hline Hispanic, $n(\%)$ & $65(56)$ & $30(50)$ & $8(57)$ \\
\hline \multicolumn{4}{|l|}{ Clinical } \\
\hline STEMI, $n(\%)$ & $14(12)$ & $11(18)$ & $1(7)$ \\
\hline NSTEMI, $n(\%)$ & $41(35)$ & $16(27)$ & $8(57)$ \\
\hline Unstable angina, $n(\%)$ & $61(53)$ & $32(54)$ & $5(36)$ \\
\hline GRACE risk score & $89.1 \pm 28.2$ & $91.5 \pm 28.1$ & $84.2 \pm 33.2$ \\
\hline $\mathrm{LVEF} \geq 40, n(\%)$ & $13(11)$ & $12(20)$ & $3(21)$ \\
\hline Charlson comorbidity index & $1.5 \pm 1.6$ & $1.9 \pm 1.8$ & $1.7 \pm 1.6$ \\
\hline $\begin{array}{l}\text { Antidepressant medication from electronic health record or } \\
\text { self-report at discharge }\end{array}$ & $0(0)$ & $0(0)$ & $8(57)$ \\
\hline \multicolumn{4}{|l|}{ Emergency department } \\
\hline ED census & $226 \pm 25$ & $230 \pm 25$ & $227 \pm 24$ \\
\hline High ED crowding, $n(\%)$ & $41(35)$ & $22(37)$ & $4(30)$ \\
\hline Medium ED crowding, $n(\%)$ & $35(30)$ & $21(36)$ & $5(35)$ \\
\hline Low ED crowding, $n(\%)$ & $40(35)$ & $16(27)$ & $5(35)$ \\
\hline \multicolumn{4}{|l|}{ Psychiatric } \\
\hline PHQ score in-hospital ${ }^{* * *}$ & $3.0 \pm 3.9$ & $4.2 \pm 4.6$ & $9.6 \pm 6.0$ \\
\hline PHQ $\geq 10, n(\%)^{* *}$ & $10(9)$ & $8(12)$ & $5(37)$ \\
\hline IES-R PTSD score 1 month ${ }^{*}$ & $4.1 \pm 8.1$ & $5.0 \pm 10.4$ & $11.7 \pm 14.9$ \\
\hline
\end{tabular}

Abbreviations: GRACE, Global Registry of Acute Coronary Events; IES-R, Impact of Events Scale-Revised; LVEF, Left ventricular ejection fraction; MI, myocardial infarction; NSTEMI, non- ST-segment elevation MI; PHQ, Patient Health Questionnaire (depression); STEMI, STsegment elevation MI

Note: Values are mean $\pm \mathrm{SD}$ unless indicated otherwise.

Note:

p $<.05$;

** $\mathrm{p}<.01$,

**** $\mathrm{p}<.001$. 